\title{
A Noise-Tolerant Group Delay Estimator Applied to Dispersion Measurement in Gases
}

\author{
J. E. Carlson ${ }^{\dagger, *}$, F. Sjöberg ${ }^{\ddagger}$, and P.-E. Martinsson ${ }^{\dagger}$ \\ ${ }^{\dagger}$ EISLAB, Dept. of Computer Science and Electrical Engineering, Luleå University of Technology, SE-971 87 Luleå, Sweden. \\ ${ }^{\ddagger}$ Div. of Signal Processing, Dept. of Computer Science and Eletrical Engineering, Luleå University of Technology, \\ SE-971 87 Luleå, Sweden \\ *Email: Johan.Carlson@sm.luth.se
}

Abstract-In this paper we present a model-based group velocity estimator, that can be used to measure speed of sound in ultrasonic pulse-echo systems as a function of ultrasound frequency. The estimation of group velocities involves numerical differentiation of the phase difference. In the presence of noise, this becomes numerically unstable. The model-based approach presented herein, shows better tolerance to experimental noise.

The performance of the estimator is evaluated with simulations as function of pulse bandwidth and SNR.

Finally, the estimator applied to real data and compared with other methods for measuring speed of sound in Ethane and Oxygen.

\section{INTRODUCTION}

When speed of sound is measured with pulse-echo ultrasound, the most common method is to use a standard crosscorrelation. An echo that have propagated a known distance is cross-correlated with a reference echo, and the maximum of the cross-correlation function corresponds to the timedelay between the measured echo and the reference echo. This works well if the shape of the reflected echo is the same as the shape of the reference echo. If, however, the shape of the pulse has changed, due to frequency dependent attenuation, or frequency dependent sound velocity (i.e. dispersion), the cross-correlation method will give a biased estimate.

If we are interested only in an average value of the speed of sound (looking at the entire pulse) a better way than the standard cross-correlation method, would be to use the analytic cross-correlation function presented by Marple [1].

In this paper we present and evaluate a method to estimate sound velocity as function of the frequencies present in the ultrasound pulse. The performance of the estimator is then evaluated with simulations and with experiments on gases (Oxygen and Ethane). The group velocity measurements are compared with the standard cross-correlation method, as well as values obtained with Marple's method.

\section{THEORY}

This section describes the general principle of estimating the group delay, as well as the model-based approach proposed in this paper. All derivations are made with the continuous-time representation, but the modifications needed when working with sampled versions are straightforward.

\section{A. Estimating Group Delay}

Let $x_{1}(t)$ and $x_{2}(t)$ be two ultrasound pulses, where $x_{1}(t)$ is a reference echo, and $x_{2}(t)$ is an attenuated and delayed version of $x_{1}(t)$, that have propagated a known distance, $\Delta x$, through a medium with unknown acoustic properties.

If we assume that the sound propagation through the medium can be modelled as a linear system described by the impulse response, $h(t)$, then

$$
x_{2}(t)=x_{1}(t) * h(t)=\int_{-\infty}^{\infty} x(\tau) h(t-\tau) d \tau
$$

where $(*)$ denotes convolution. Let $X_{1}(\omega), X_{2}(\omega)$, and $H(\omega)$ be the Fourier-transforms of the echoes and the impulse response of the medium. Then, in the frequency domain, Eq. (1) corresponds to

$$
X_{2}(\omega)=X_{1}(\omega) H(\omega)=A(\omega) X_{1}(\omega) e^{j \phi(\omega)},
$$

where $\omega$ represents angular frequencies $(\mathrm{rad} / \mathrm{s})$, and where $A(\omega)$ and $\phi(\omega)$ are the magnitude and phase of $H(\omega)$, respectively.

The group delay, $\psi(\omega)$, is defined as the derivative of the phase difference $\phi(\omega)$, with respect to the angular frequency, $\omega$, that is

$$
\psi(\omega) \triangleq-\frac{\partial}{\partial \omega} \tilde{\phi}(\omega)
$$

where $\tilde{\phi}(\omega)$ is the unwrapped version of the phase difference 
$\phi(\omega)$ [2], given by

$$
\begin{aligned}
& \phi(\omega)=\arg H(\omega)=\arg \left(\frac{X_{2}(\omega)}{X_{1}(\omega)}\right)= \\
& \tan ^{-1}\left(\frac{\operatorname{Im}\left\{X_{2}\right\}}{\operatorname{Re}\left\{X_{2}\right\}}\right)-\tan ^{-1}\left(\frac{\operatorname{Im}\left\{X_{1}\right\}}{\operatorname{Re}\left\{X_{1}\right\}}\right) \pm n 2 \pi .
\end{aligned}
$$

The $n 2 \pi$ ambiguity of $\phi(\omega)$ is due to the $\tan ^{-1}$ function. This becomes a problem when working with phase velocity measurements, as recognized by Löfqvist [3].

From the propagation distance $\Delta x$ and the group delay in Eq. (3), we obtain the group velocity $c(\omega)$ as

$$
c(\omega)=\Delta x / \psi(\omega)
$$

which is the speed of sound through the medium, as a function of ultrasound frequency $\omega$.

If the experimental noise results in phase jumps of more than $\pi$, it will affect the performance of the noiseunwrapping algorithm used to obtain the phase difference. It is not, however, affected by the $n 2 \pi$ ambiguity associated with phase delay measurement, since this term vanishes during the differentiation.

In a non-dispersive medium, i.e. when the speed of sound does not depend on frequency, the group delay is constant. It might, however, still be a different value than that obtained using a standard cross-correlation, if the acoustic attenuation in the medium is frequency-dependent.

\section{B. Model-Based Approach}

As described in the previous section, estimating the group delay involves a differentiation of the phase curve with respect to the angular frequency, $\omega$. In the presence of noise, this differentiation becomes numerically unstable due to noise amplification.

In this paper we propose an alternative technique, based on the differentiation of a parametric model of the phase difference. Assume, for example, that within the bandwidth of the pulse, the unwrapped phase difference, $\tilde{\phi}(\omega)$, can be approximated as

$$
\hat{\phi}(\omega)=f(\omega) \approx a_{0}+a_{1} \omega+a_{2} \omega^{2} .
$$

The model parameters $a_{0}, a_{1}$, and $a_{2}$ can then easily be determined using a least-squares fit to the measured phasedelay. The model, $\hat{\phi}(\omega)$ can then be differentiated either analytically or numerically.

A method like this is affected by two main sources of error:

1. Lack-of-fit, i.e. modelling error (Bias).

2. Experimental noise (Random).

The model error is the more difficult to address. To test if a given model is appropriate, the standard method is to study the residuals [4]. The residuals should be uncorrelated to the measured values.

One could assume a high-order polynomial model, and obtain reasonably good results. An even better way, if possible, is to use a priori knowledge of the underlying physics. If no physical model is available, different empirical models (polynomial, exponential, etc.) can be tested against each other, using for example Analysis of Variance (ANOVA) [4].

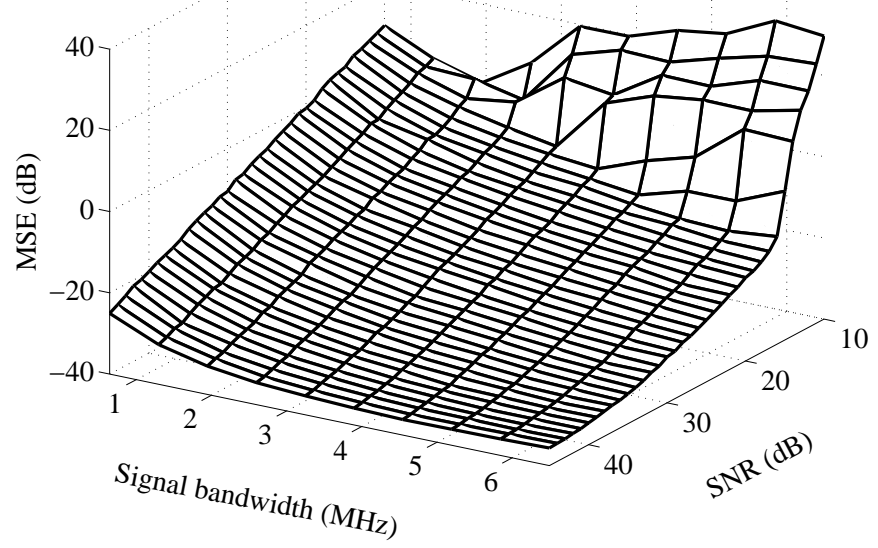

Fig. 1. MSE of group delay error when using a 2nd degree polynomial fit to the unwrapped phase curve.

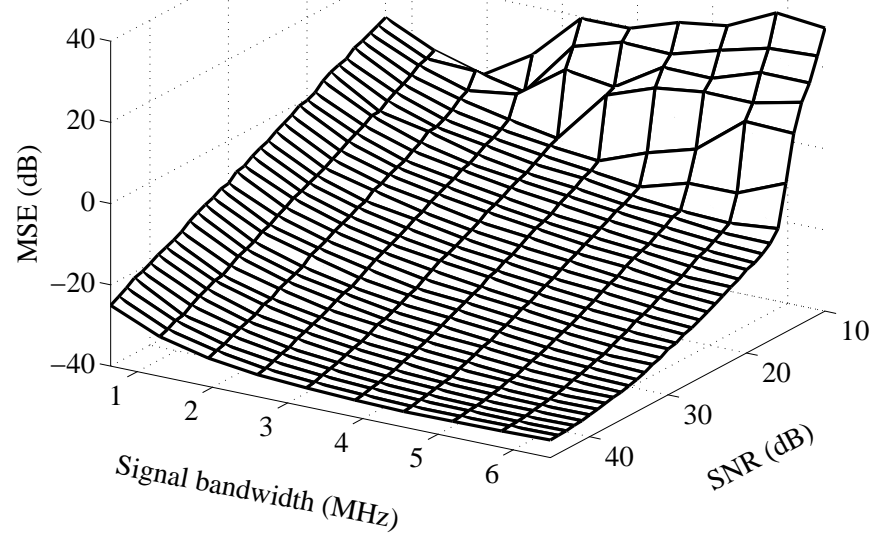

Fig. 2. MSE of group delay error when using a 3rd degree polynomial fit to the unwrapped phase curve.

\section{Simulations}

In this section the performance of the estimator in Section II-B is evaluated with respect to pulse bandwidth and 
signal-to-noise ratio (SNR). The analysis is based on simulated data, and the estimator is then applied to real experimental data, to estimate dispersion effects in gases.

\section{A. Design}

The simulations are performed in the following way. A chirp-signal is sent through a linear time invariant filter, which models the frequency dependent group velocity, and white Gaussian noise is added to the received signal. We measure the performance of the estimator as mean square error (averaged over realizations of the simulations and over pulse bandwidth) for different values of SNR and bandwidth of the chirp.

The sampling frequency is $20 \mathrm{MHz}$, and the chirp signal is 4096 samples long and has linearly increasing frequency. A raised-cosine window with roll-off factor 0.7 is applied to smoothen the envelope of the chirp. In the simulations we define the bandwidth of the pulse as where the magnitude has dropped $3 \mathrm{~dB}$.

An impulse response is generated, which has a transfer function with essentially flat amplitude, and a phase that gives a desired group velocity. The group velocity is here modelled to increase linearly from 300 to $310 \mathrm{~m} / \mathrm{s}$ in the frequency range 0 to $10 \mathrm{MHz}$. This model results in an almost linear phase difference, which implies that a second order polynomial model of the unwrapped phase should work fine.

\section{B. Performance Analysis}

Figures 1 and 2 show the mean square error (MSE) of the group velocity for the estimator using a second order polynomial and a third order polynomial model, respectively. In general, larger signal bandwidth yields better velocity estimation. Also, the second order model is slightly better than the third order model, which is reasonable, since this is closer to the true phase. The third order model tends to over model the phase, and therefore becomes more sensitive to noise. The increase in error variance seen for low SNR and larger bandwidths is due to errors in the unwrapping of the phase.

We can also see that for very large SNR, the MSE increases slightly for larger bandwidths. This is due to the fact that the residual model mismatch, which dominates the MSE for high SNR, increases with bandwidth.

\section{Gas Measurements}

\section{A. Experimental Setup and Design}

A pulse echo measurement scheme as shown in Fig. 3 was used to measure the speed of sound of Oxygen and Ethane. A custom-built pressure chamber (see Fig. 3) was used to achieve different static pressures. For each gas, the pressure was varied from 1.0 to 7.4 bar in 19 steps.

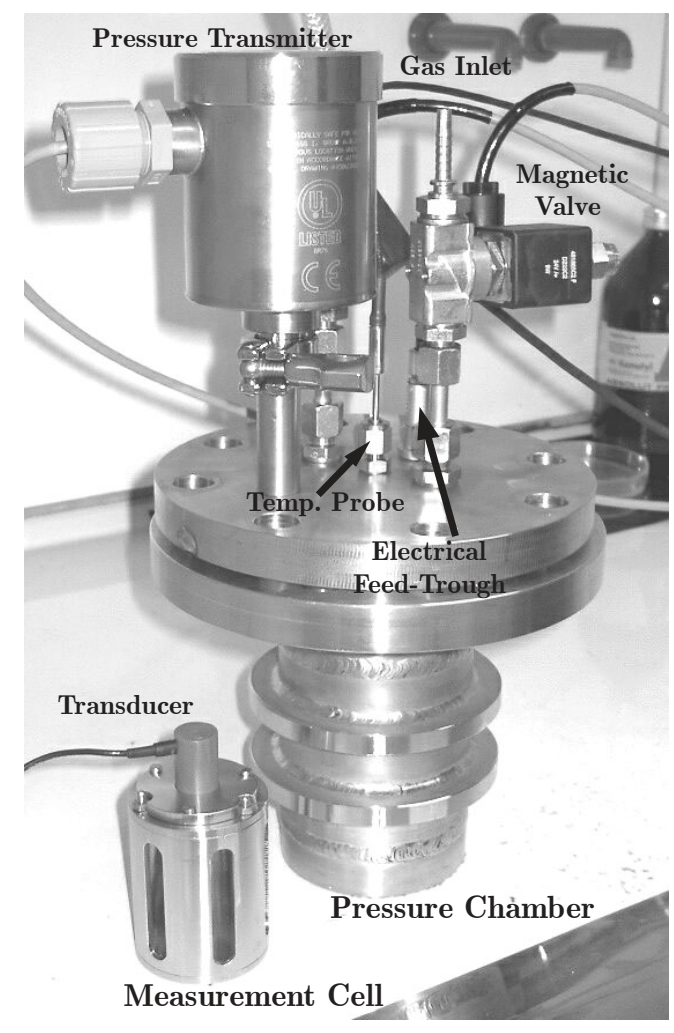

Fig. 3. The measurement equipment. All measurements were performed in a pressure chamber. The pressure was varied between 1.0 bar and 7.4 bar. The air transducer had a center frequency of $1 \mathrm{MHz}$.

The transducer was mounted on a stainless steel measurement cell, as seen in the lower left corner of Fig. 3. The measurement cell was then immersed into the pressure chamber. The whole setup was then placed in a temperature controlled chamber (Heraeus Vötsch HT4010), keeping the temperature constant.

The pressure in the chamber was measured with an $A N$ DERSON TPP Pressure Transmitter. The transmitter has an accuracy of approximately 30 mbar, with a range of up to 13.6 bar above atmospheric zero.

The ultrasound transducer was connected to a Panametrics Pulser/Receiver Model 5072. This pulser/receiver is able to both excite the transducer, and to amplify the received echoes.

All pulses were sampled at $100 \mathrm{MHz}$ with a Tektronix TDS 724, digitizing oscilloscope. For each experimental setting, 50 pulses were recorded and transferred to a PC, where they were averaged and further processed.

\section{B. Results}

Fig. 4 shows the measured speed of sound in Ethane, at $20{ }^{\circ} \mathrm{C}$, for a $\mathrm{f} / \mathrm{P}$ interval of $100-600 \mathrm{kHz} / \mathrm{bar}$. The results obtained with the standard cross-correlation, Marple's 
method, and our group velocity estimator agree fairly well. This is the case because the ultrasound pulse is narrowband, and therefore the dispersion does not affect the pulse shape much.

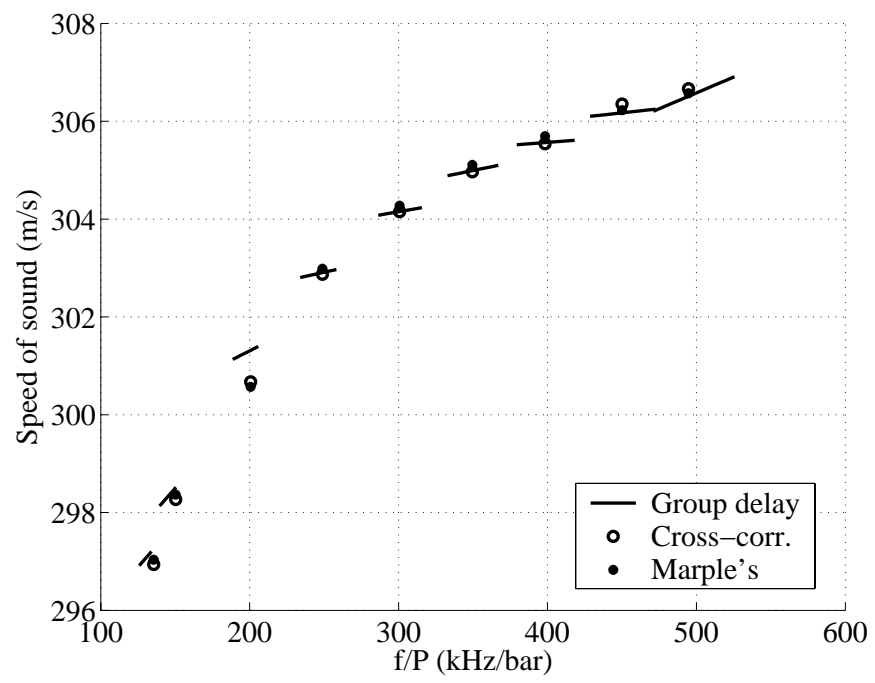

Fig. 4. Speed of sound in Ethane, measured at $20^{\circ} \mathrm{C}$, comparing results of using a standard cross-correlation method, Marple's method, or the group delay estimator proposed in this paper.

Fig. 5 shows the measured speed of sound in Oxygen, also at $20{ }^{\circ} \mathrm{C}$, for a f/P interval of $100-700 \mathrm{kHz} / \mathrm{bar}$. It is clear from the measurement that Oxygen is less dispersive than Ethane. All three estimators still give similar results.

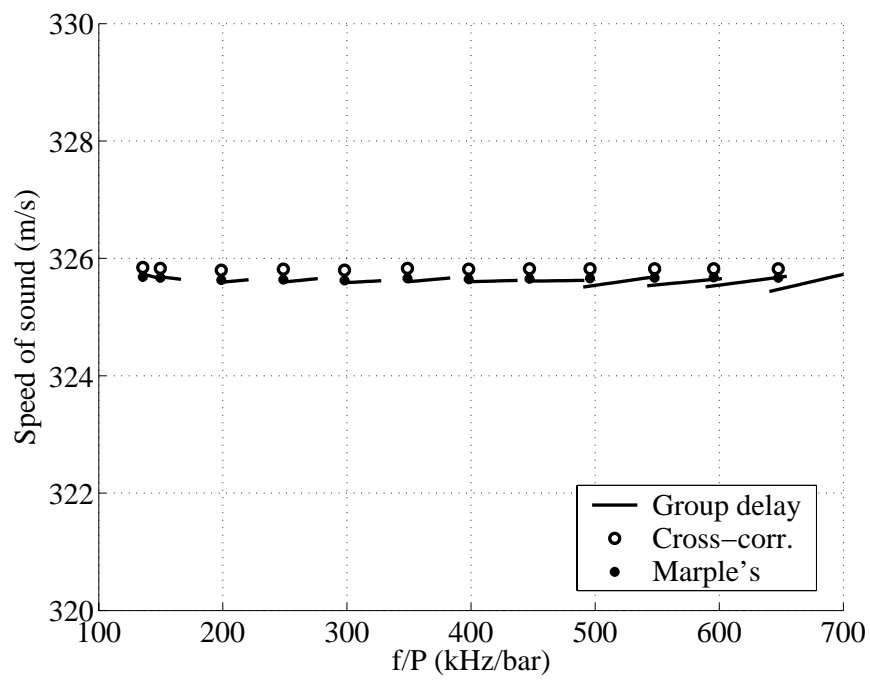

Fig. 5. Speed of sound in Oxygen, measured at $20^{\circ} \mathrm{C}$, comparing results of using a standard cross-correlation method, Marple's method, or the group delay estimator proposed in this paper.

\section{DISCUSSION}

The single most important step in the procedure described in this paper, is to choose a good model for the phase differ- ence. Good knowledge of the underlying physics can help finding a model for the speed of sound. This is then easily converted to a model of the phase difference. The phase model might not be a polynomial model, where the coefficients can be found by a simple linear least-squares fit. For a model to be valid in a wide frequency range, it will most likely be necessary to use non-linear, iterative numerical method to fit the model to the measured phase difference. It should be noted, however, that for a limited pulse bandwidth, a simple model may often be sufficiently accurate. For a wide pulse bandwidth, using a spline fit might give satisfying results.

As seen in the upper right corner of Figures 1 and 2, the current algorithm does not work well when for low SNR. The reason for this is not the numerical differentiation in Eq. (3), but the phase unwrapping performed prior to the differentiation. The phase unwrapping algorithm we used in this paper is too sensitive to noisy data. Future work will involve improving the noise unwrapping stage, possibly using the method proposed by Tribolet [5] or by Gough [6].

\section{CONCLUSIONS}

In this paper we propose a model-based group velocity estimator, useful for example in ultrasonic dispersion measurement of gases.

The estimator is evaluated for different signal bandwidth and for different SNR. Simulation results show that, given that the phase unwrapping algorithm works, the estimator works properly.

Finally, the estimator is used to measure frequency dependent sound velocity (dispersion) in Ethane and Oxygen. The results agree well with those obtained with standard crosscorrelation. This is because the pulses used in the experiments were fairly narrowband. For larger pulse bandwidth, the dispersion would cause the cross-correlation techniques to give biased results.

\section{REFERENCES}

[1] S. L. Marple, Jr., "Estimating Group Delay and Phase Delay via Discrete-Time "Analytic" Cross-Correlation," IEEE Trans. Sig. Proc., vol. 47, no. 9, pp. 2604-2607, 1999.

[2] A. V. Oppenheim and R. W. Shafer, Discrete-Time Signal Processing. Englewood Cliffs, N.J.: Prentice Hall, 1989.

[3] T. Löfqvist, "Ultrasonic Wave Attenuation and Phase Velocity in a Paper-Fibre Suspension," in Proc. IEEE Int. Ultrason. Symp., (Toronto, Canada), pp. 841-844, 1997.

[4] G. E. P. Box and N. R. Draper, Empirical Model-Building and Response Surfaces. New York: Wiley Interscience, 1987.

[5] J. M. Tribolet, "A New Phase Unwrapping Algorithm," IEEE Trans. Acoust., Speech, and Sig. Proc., vol. ASSP-25, pp. 170-177, April 1977.

[6] P. T. Gough, "A Particular Example of Phase Unwrapping Using Noisy Experimental Data," IEEE Trans. Acoust., Speech, and Sig. Proc., vol. ASSP-31, pp. 742-744, June 1983. 